\title{
Surface-Electrode Rydberg-Stark Decelerator
}

\author{
S. D. Hogan, P. Allmendinger, H. Saßmannshausen, H. Schmutz, and F. Merkt \\ Laboratorium für Physikalische Chemie, ETH Zürich, CH-8093, Switzerland
}

(Received 25 October 2011; published 10 February 2012)

\begin{abstract}
Hydrogen atoms in Rydberg states with principal quantum numbers between 23 and 70 have been accelerated, decelerated, and electrostatically trapped using a surface-electrode Rydberg-Stark decelerator. By applying a set of oscillating electrical potentials to a two-dimensional array of electrodes on a printed circuit board (PCB), a continuously moving, three-dimensional electric trap with a predefined velocity and acceleration is generated. From an initial longitudinal velocity of $760 \mathrm{~m} / \mathrm{s}$, final velocities of the Rydberg atoms ranging from $1200 \mathrm{~m} / \mathrm{s}$ to zero velocity in the laboratory-fixed frame of reference were achieved. Accelerated or decelerated atoms were detected directly by pulsed electric-field ionization. Atoms trapped at zero mean velocity above the PCB were reaccelerated off the PCB before field ionization.
\end{abstract}

Controlled manipulation of the motional degrees of freedom and internal quantum states of atoms and molecules at vacuum-solid-state interfaces is of importance in several areas of research. Robust and scalable chip-based electric and magnetic traps and guides have been developed for atomic ions [1] and neutral ground state atoms [2]. These devices have been exploited, e.g., in quantum information processing [3] and quantum metrology [4]. In addition, the preparation of cold, velocity-controlled samples of polar molecules using chip-based Stark decelerators has been demonstrated as a route "towards a gas phase molecular laboratory on a chip" $[5,6]$. Approaches directed toward the confinement of Rydberg atoms in the vicinity of surfaces have involved Rydberg photoexcitation in miniature vapor cells [7], and in close proximity to arrays of surfacebased permanent-magnet traps [8]. Atom chips have also been developed with Rydberg photoexcitation in the strong dipole-blockade regime in mind $[9,10]$, for the realization of sources of single atoms on demand [11].

We report here the acceleration, deceleration, and electrostatic trapping of hydrogen Rydberg atoms using a surface-electrode Rydberg-Stark decelerator. The possibility of transporting Rydberg atoms or molecules above a surface in continuously moving electric traps which is demonstrated, opens up a range of opportunities relevant to studies of Rydberg-atom- or Rydberg-molecule-surface interactions [12,13] and, in combination with chip-based superconducting microwave circuits [14], to hybrid approaches to cavity quantum electrodynamics [15]. Together with excitation in the strong dipole-blockade regime, one can also envisage the transport of single-atoms on demand, or the preparation of arrays of trapped Rydberg atoms for applications in quantum simulation and quantum information processing.

The decelerator exploits the large electric dipole moments associated with Rydberg-Stark states of high principal quantum number $n$, and the forces which they experience in the presence of inhomogeneous electric fields. These dipole moments are equal to $\frac{3}{2} n k e a_{0}$, where $k$ takes values from $-(n-|m|-1)$ to $+(n-|m|-1)$ in steps of two [16]. Following early proposals by Breeden and Metcalf [17], and Wing [18], the first experimental work to exploit such forces on samples in Rydberg states involved the use of inhomogeneous electric fields to deflect beams of krypton atoms [19] and decelerate $\mathrm{H}_{2}$ [20] and argon [21]. This later led to the three-dimensional electrostatic trapping of $\mathrm{H}[22,23]$ and $\mathrm{H}_{2}[24,25]$. The electrode configurations used in these experiments were single-stage devices which acted as both decelerators and traps, with continuously moving electric-field minima generated to provide full three-dimensional confinement during deceleration and trapping.

The aspects of the surface-electrode-based decelerators described here which distinguish them from the singlestage devices used in previous work [22-25] include their scalability and flexibility with regard to the preparation of a wide range of electrode geometries, and the possibility to achieve efficient deceleration using large electric-field gradients but weak fields. Deceleration in weak fields reduces undesirable losses arising from field ionization at high $n$ and considerably increases the range of states that can be decelerated. Several benefits result: (i) at a particular temperature, a value of $n$ can be chosen for which the blackbody-radiation-induced depopulation rate is lowest; (ii) for molecules with small rotational constants, deceleration can be performed in weak fields, at a position in the Rydberg series where interactions between states converging to different rotational levels of the ion core are minimal; (iii) the lifetimes of Rydberg states rapidly increase with $n$; and (iv) the opportunity to decelerate Rydberg atoms and molecules with $n>50$ and electric dipole moments $>9000$ Debye makes the deceleration of a wide range of species possible.

The experiments involve the production of $\mathrm{H}$ atoms by $193 \mathrm{~nm}$ laser photolysis of $\mathrm{NH}_{3}$ in a quartz capillary mounted at the exit of a pulsed valve [26]. $\mathrm{NH}_{3}$ is seeded 
in argon at a ratio of 1:9 and the $\mathrm{H}$ atoms in the beam have a mean longitudinal velocity of $760 \mathrm{~m} / \mathrm{s}$. After passing a skimmer, the atoms enter a pair of circular metallic plates separated by $10 \mathrm{~mm}$ with their surfaces perpendicular to the axis of propagation of the beam [see Fig. 1(a)]. These electrodes are used to generate a homogeneous electric field of $33 \mathrm{~V} / \mathrm{cm}$ in which the $1^{2} S$ ground state $\mathrm{H}$ atoms are photoexcited to low-field-seeking Rydberg-Stark states in a resonant two-color (vacuum ultraviolet + ultraviolet), two-photon transition via the $2{ }^{2} P$ state. The Rydberg atoms then travel out of the excitation region and over the surface-electrode decelerator in the positive $z$ direction. After passing over the decelerator the atoms are detected by pulsed electric-field ionization $\sim 3.5 \mathrm{~mm}$ beyond the printed circuit board (PCB), between a second pair of circular metallic plates. The resulting $\mathrm{H}^{+}$ions are detected on a microchannel-plate (MCP) detector.

The principle of the decelerator is similar to that used by Meek et al. [5,27] for the deceleration of $\mathrm{CO}$ molecules, but adapted to deceleration in Rydberg states. The width of

(a)

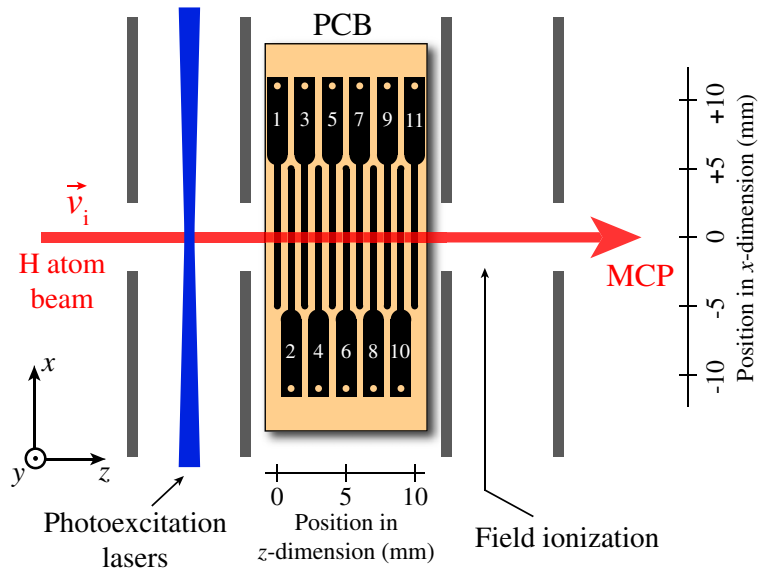

(b)

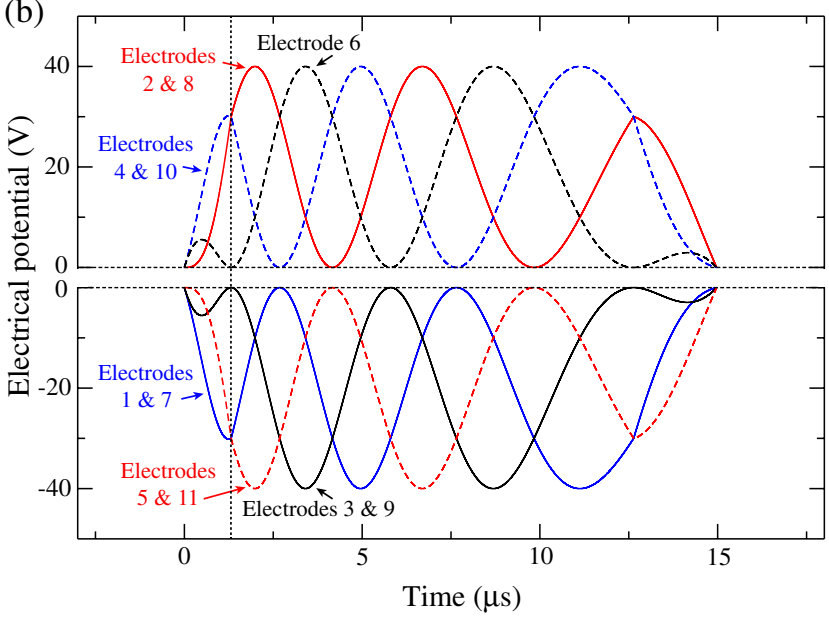

FIG. 1 (color online). (a) Schematic diagram of the surfaceelectrode-based Rydberg-Stark decelerator and surrounding photoexcitation and field-ionization regions. (b) Oscillating potentials applied to the 11 electrodes for deceleration from $v_{i}=$ $760 \mathrm{~m} / \mathrm{s}$ to $v_{f}=300 \mathrm{~m} / \mathrm{s}$.
$0.5 \mathrm{~mm}$ and center-to-center spacing $d_{z}=1.0 \mathrm{~mm}$ between the 11 electrodes in the $z$ dimension was chosen to match the decelerator acceptance to the phase-space characteristics of the Rydberg-atom beam. The ends of the electrodes in the $x$ dimension have been enlarged to reduce the oscillatory motion of the minima in this dimension during deceleration. The decelerator is operated by applying six different oscillating electrical potentials $V_{i}$ to electrodes numbered $i=1-6$ in Fig. 1(a) and repeating the sequence from the seventh electrode on. These potentials (called waveforms hereafter) have the general form $V_{i}=$ $(-1)^{i} V_{0}\left[1+\cos \left(\omega t+\phi_{i}\right)\right]$, where $2 V_{0}$ is the peak-topeak amplitude, $\omega$ is the oscillation frequency, and $\phi_{i}=$ $(i-1) 2 \pi / 3$ [5]. Using this configuration, a set of moving electric traps, separated by a distance of $3 d_{z}=3 \mathrm{~mm}$, are generated above the PCB as depicted in Fig. 2. The switchon time of the waveforms is selected to ensure that all Rydberg atoms prepared are loaded into a single trap. As the potentials oscillate, this trap moves in the positive $z$ dimension with a velocity $v_{z}=3 d_{z} \omega /(2 \pi)$. Acceleration or deceleration, $a_{z}$, is achieved by applying a linear chirp to the waveforms such that $\omega(t)=\omega_{0}+\left(2 \pi / 3 d_{z}\right) a_{z} t$ where $\omega_{0}$ corresponds to the initial velocity $v_{i}$. An example of a set of waveforms for deceleration from $v_{i}=760 \mathrm{~m} / \mathrm{s}$ to a final velocity $v_{f}=300 \mathrm{~m} / \mathrm{s}$ is presented in Fig. 1(b). The waveforms are switched on only when the atoms are above the PCB to avoid undesirable heating by the gradients of the fringe fields at the edges of the PCB. During switch-on and -off $\omega$ remains constant and $V_{0}$ is ramped linearly.
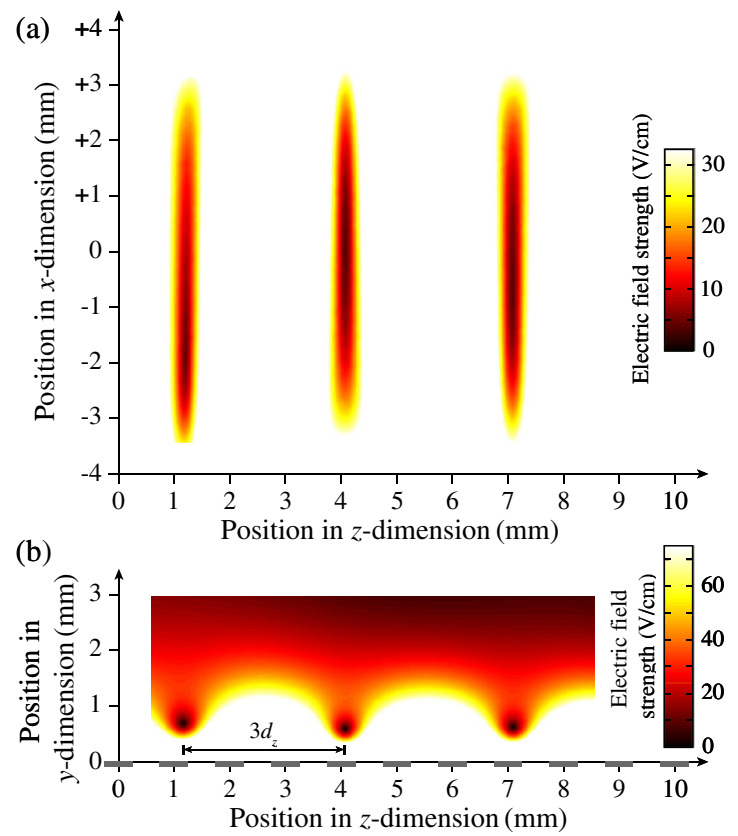

FIG. 2 (color online). Electric-field distribution in (a) the $x z$ plane containing the trap minima and (b) the $x=0$ plane. The positions of the decelerator electrodes are indicated on the horizontal axis in (b). The $0 \mathrm{~V}$ plate is located at $y=-0.75 \mathrm{~mm}$. 
The potentials used to generate the field distributions depicted in Fig. 2 correspond to those at a time of $1.25 \mu \mathrm{s}$ in Fig. 1(b), at the end of the switch-on phase (dotted vertical line). For the value of $V_{0}=20 \mathrm{~V}$ used here, the trap minimum into which the atoms to be decelerated are loaded is located $0.6 \mathrm{~mm}$ above the surface of the PCB in the $y$ dimension. The moving trap has been chosen to be $\sim 5 \mathrm{~mm}$ long in the $x$ dimension and $\sim 0.5 \mathrm{~mm}$ wide in the $y$ and $z$ dimensions. The trap depth is $\sim 27 \mathrm{~V} / \mathrm{cm}$, corresponding $\sim 2 \mathrm{~K}(\sim 5.5 \mathrm{~K})$ for the outer low-field-seeking Stark states at $n=30(n=50)$.

The operation of the decelerator was investigated by measuring $\mathrm{H}$-atom time-of-flight distributions between the position of photoexcitation and that of field ionization. A set of data corresponding to acceleration or deceleration from $v_{i}=760 \mathrm{~m} / \mathrm{s}$ to final velocities between 1200 and $200 \mathrm{~m} / \mathrm{s}$, is presented in Fig. 3(a). These measurements only differ in the chirp used to achieve the desired final velocity. The time-of-flight distribution labeled (iii) corresponds to the undecelerated beam of Rydberg atoms, detected at a time of $\sim 29 \mu$ s (vertical dotted line). This measurement was made with the decelerator off and its intensity is scaled by a factor of 0.5 with respect to the other measurements. Measurements (i) and (ii) were performed to accelerate the atoms to $v_{f}=1200$ and $1000 \mathrm{~m} / \mathrm{s}$, respectively. The accelerated atoms arrive at the detection region earlier than the undecelerated atoms, at times of $\sim 26$ and $\sim 27.5 \mu \mathrm{s}$. The top four time-of-flight distributions (iv)-(vii) correspond to deceleration to $v_{f}=$ $600,450,300$, and $200 \mathrm{~m} / \mathrm{s}$ and indicate progressively later arrival times of the decelerated atoms of 33, 38, 45, and $54 \mu \mathrm{s}$, respectively. The intensity of the distribution corresponding to deceleration to $v_{f}=200 \mathrm{~m} / \mathrm{s}$ is scaled by a factor of 4 . (a)

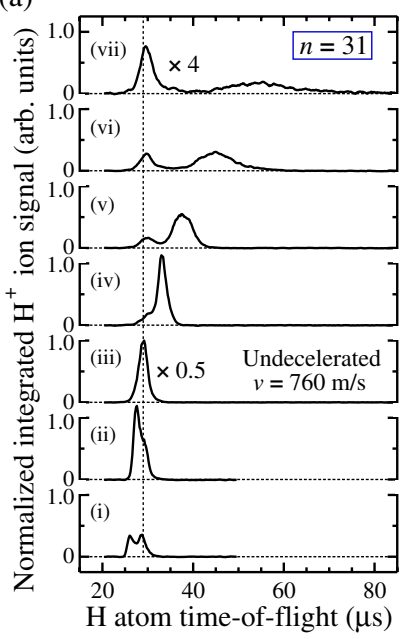

(b)

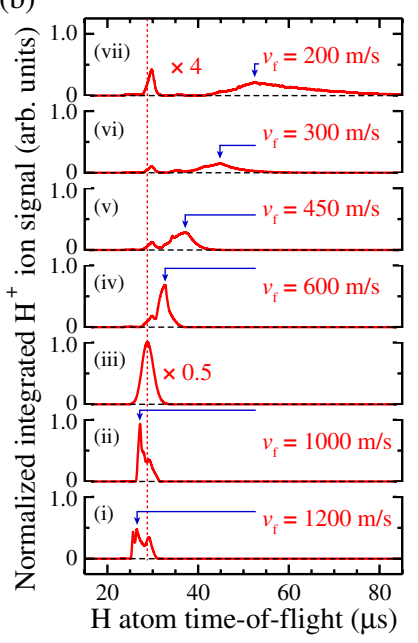

FIG. 3 (color online). (a) Experimental and (b) simulated Hatom time-of-flight distributions demonstrating acceleration or deceleration at $n=31$ from $v_{i}=760 \mathrm{~m} / \mathrm{s}$ to $v_{f}=1200,1000$, $600,450,300$, and $200 \mathrm{~m} / \mathrm{s}$.
Three-dimensional particle-trajectory simulations, presented in Fig. 3(b), capture the essential features of the experimental data and provide a basis for their interpretation. For each simulation, a random initial distribution of particles was generated corresponding to the Rydberg atoms in the photoexcitation volume. They have a full width at half maximum of $0.8 \mathrm{~mm}$ in the $y$ and $z$ dimensions and $4 \mathrm{~mm}$ in the $x$ dimension. The mean longitudinal velocity was $760 \mathrm{~m} / \mathrm{s}$ with a relative velocity spread $\sigma_{v_{z}}$ characterized by a longitudinal temperature $T_{z}=$ $\frac{1}{2} m_{\mathrm{H}} \sigma_{v_{z}}^{2} / k_{\mathrm{B}}=110 \mathrm{mK}$, where $m_{\mathrm{H}}$ is the mass of the $\mathrm{H}$ atom and $k_{\mathrm{B}}$ is the Boltzmann constant, as determined from time-of-flight measurements of the Rydberg-atom beam. The transverse temperatures were $T_{x}=75 \mathrm{mK}$ and $T_{y}=$ $5 \mathrm{mK}$, resulting from the intersection of the laser beams propagating in the $x$ dimension with the molecular beam.

The quantitative agreement between the measured and calculated time-of-flight distributions in Fig. 3 enables the complete characterization of the experimental results: The peak observed in all traces at $\sim 29 \mu$ s corresponds to atoms which traverse the PCB at a distance larger than the saddle point of the trap in the $y$ dimension (i.e., at $y>$ $1.2 \mathrm{~mm}$ ). These atoms follow metastable trajectories across the decelerator and arrive at the field-ionization point without being accelerated or decelerated. The second peak corresponds to the accelerated or decelerated atoms. The velocities at the peak maxima [indicated by arrows in Fig. 3(b)] match the final velocities for which the waveforms were designed. At low final velocities, the bunch of decelerated atoms expand in the longitudinal and transverse dimensions in the time between the switch off of the decelerator and detection. Transverse expansion after deceleration leads to a reduction in detection efficiency which is also reproduced by the simulations.

Compared to previous Rydberg-Stark deceleration experiments, the Rydberg atoms decelerated in the present study experience lower electric fields, because the speed at which the trap moves exactly matches the speed of the Rydberg-atom cloud at all times. Consequently, the decelerator can be used for states of higher principal quantum number. This is illustrated by the spectrum displayed in Fig. 4 which was recorded by monitoring the signal of atoms decelerated to $300 \mathrm{~m} / \mathrm{s}$ as a function of the excitation wave number relative to the field-free ionization threshold. The spectrum indicates that deceleration can be performed in the range of $n$ between 23 and $\sim 70$. The Stark states most efficiently decelerated all lie to the highenergy side of the field-free positions indicated by vertical lines and are low-field-seeking states, as expected. The electric field at which the outermost low-field-seeking Stark state at $n=70$ ionizes is $\sim 24 \mathrm{~V} / \mathrm{cm}$. The proximity of this value to the field of $\sim 27 \mathrm{~V} / \mathrm{cm}$ at the saddle point of the moving trap indicates that the high- $n$ cutoff in decelerator acceptance results from field ionization during deceleration. 


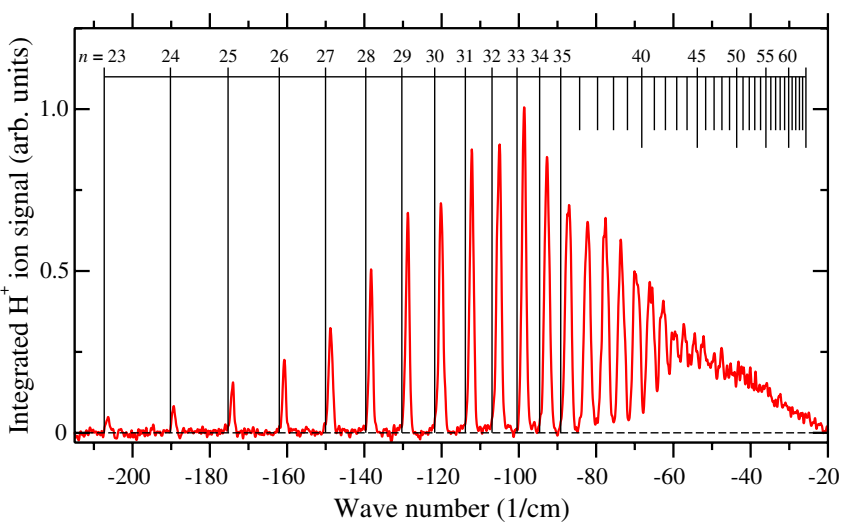

FIG. 4 (color online). Spectrum demonstrating the range of Rydberg states that can be decelerated from $v_{i}=760 \mathrm{~m} / \mathrm{s}$ to $v_{f}=300 \mathrm{~m} / \mathrm{s}$. The field-free positions of the $n=23-65$ Rydberg states below the series limit are indicated by vertical lines.

The detection efficiency of Rydberg atoms with final velocities below $300 \mathrm{~m} / \mathrm{s}$ is limited by the longitudinal and transverse expansion of the decelerated atom cloud between the end of the decelerator and the field-ionization point (see Fig. 3). However, electrostatic trapping of the Rydberg atoms above the PCB can be studied by deceleration to zero longitudinal velocity, followed by reacceleration off the PCB toward the field-ionization region. A trapping time of variable duration, $\tau_{\text {trap }}$, during which the potentials on the decelerator electrodes are held constant, is inserted between deceleration and reacceleration. The results of a set of measurements of this kind are presented in Figs. 5(a)-5(c) for $\tau_{\text {trap }}=10,20$, and $40 \mu \mathrm{s}$, respectively. The differences in times of flight correspond exactly to the differences in trapping time. The reduction in the signal intensity over this range of trapping times, if assumed to be exponential, has a time constant of $\sim 30 \mu \mathrm{s}$. Because the atoms are trapped on the axis of the molecular beam in these measurements, trap loss at early times is dominated by collisions with the trailing edge of the molecular beam. This conclusion is supported by previous measurements at similar beam densities in a threedimensional electrostatic trap which revealed collisional losses at early times, with time constants of $\sim 40 \mu \mathrm{s}$ [22]. These losses could be avoided by implementing an off-axis trap as demonstrated by Seiler et al. [23].

The surface-electrode decelerator presented here can be scaled up by placing additional electrodes on the PCB and represents an attractive way to decelerate heavier species. The distance between the trap minima and the surface can be adjusted by changing the electrode dimensions or adjusting the potentials, opening up new opportunities in the study of Rydberg-atom-surface interactions. By combining this decelerator with other surface-based devices to manipulate the internal quantum states, such as microwave waveguides [28] and resonators, a complete experimental

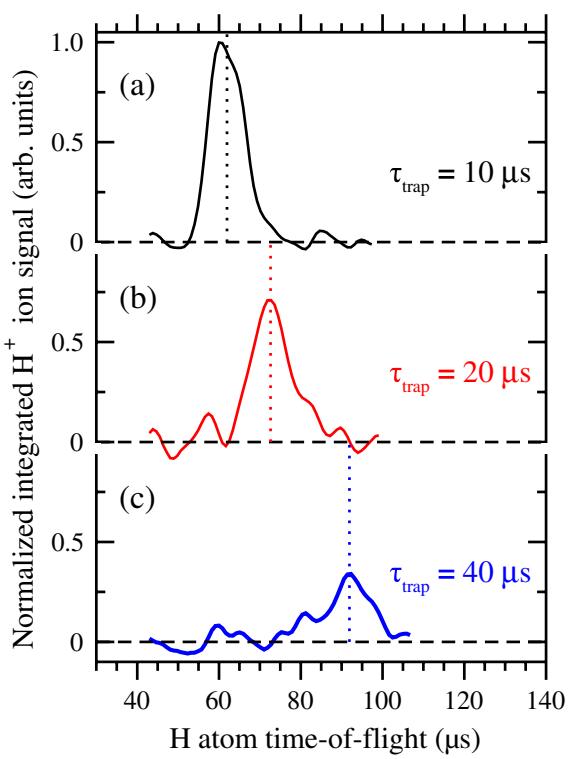

FIG. 5 (color online). H-atom time-of-flight distributions recorded after electrostatic trapping followed by reacceleration off the PCB for (a) $\tau_{\text {trap }}=10 \mu \mathrm{s}$, (b) $\tau_{\text {trap }}=20 \mu \mathrm{s}$, and (c) $\tau_{\text {trap }}=40 \mu \mathrm{s}$.

architecture for the control of the internal quantum states and translational degrees of freedom of Rydberg atoms and molecules close to surfaces is foreseen. Such developments have potential applications in hybrid approaches to cavity quantum electrodynamics and quantum information processing.

This work is supported by the Swiss National Science Foundation under Project No. 200021-113886 and by the European Research Council advanced grant program under Project No. 228286.

[1] J. Chiaverini, R. B. Blakestad, J. Britton, J. D. Jost, C. Langer, D. Leibfried, R. Ozeri, and D. J. Wineland, Quantum Inf. Comput. 5, 419 (2005).

[2] R. Folman, P. Kroger, J. Schmiedmayer, J. Denschlag, and C. Henkel, Adv. At. Mol. Opt. Phys. 48, 263 (2002).

[3] J.P. Home, D. Hanneke, J. D. Jost, J. M. Amini, D. Leibfried, and D. J. Wineland, Science 325, 1227 (2009).

[4] M. F. Riedel, P. Böhi, Y. Li, T. W. Hänsch, A. Sinatra, and P. Treutlein, Nature (London) 464, 1170 (2010).

[5] S. A. Meek, H. L. Bethlem, H. Conrad, and G. Meijer, Phys. Rev. Lett. 100, 153003 (2008).

[6] S. A. Meek, G. Santambrogio, H. Conrad, and G. Meijer, J. Phys. Conf. Ser. 194, 012063 (2009).

[7] H. Kübler, J. P. Shaffer, T. Baluktsian, R. Löw, and T. Pfau, Nature Photon. 4, 112 (2010).

[8] A. Tauschinsky, R. M. T. Thijssen, S. Whitlock, H. B. van Linden van den Heuvell, and R. J. C. Spreeuw, Phys. Rev. A 81, 063411 (2010).

[9] T. Nirrengarten, A. Qarry, C. Roux, A. Emmert, G. Nogues, M. Brune, J.-M. Raimond, and S. Haroche, Phys. Rev. Lett. 97, 200405 (2006). 
[10] O. Cherry, J. D. Carter, and J. D. D. Martin, Can. J. Phys. 87, 633 (2009).

[11] M. Saffman and T. G. Walker, Phys. Rev. A 66, 065403 (2002).

[12] S. B. Hill, C. B. Haich, Z. Zhou, P. Nordlander, and F. B. Dunning, Phys. Rev. Lett. 85, 5444 (2000).

[13] E. So, M. Dethlefsen, M. Ford, and T. P. Softley, Phys. Rev. Lett. 107, 093201 (2011).

[14] A. Wallraff, D. I. Schuster, A. Blais, L. Frunzio, R.-S. Huang, J. Majer, S. Kumar, S. M. Girvin, and R. J. Schoelkopf, Nature (London) 431, 162 (2004).

[15] M. Saffman, T. G. Walker, and K. Mølmer, Rev. Mod. Phys. 82, 2313 (2010).

[16] T.F. Gallagher, Rydberg Atoms (Cambridge University Press, Cambridge, England, 1994).

[17] T. Breeden and H. Metcalf, Phys. Rev. Lett. 47, 1726 (1981).

[18] W. H. Wing, Phys. Rev. Lett. 45, 631 (1980).

[19] D. Townsend, A. L. Goodgame, S.R. Procter, S.R. Mackenzie, and T. P. Softley, J. Phys. B 34, 439 (2001).
[20] S. R. Procter, Y. Yamakita, F. Merkt, and T. P. Softley, Chem. Phys. Lett. 374, 667 (2003).

[21] E. Vliegen, H. J. Wörner, T. P. Softley, and F. Merkt, Phys. Rev. Lett. 92, 033005 (2004).

[22] S. D. Hogan and F. Merkt, Phys. Rev. Lett. 100, 043001 (2008).

[23] Ch. Seiler, S. D. Hogan, H. Schmutz, J. A. Agner, and F. Merkt, Phys. Rev. Lett. 106, 073003 (2011).

[24] S. D. Hogan, Ch. Seiler, and F. Merkt, Phys. Rev. Lett. 103, 123001 (2009).

[25] Ch. Seiler, S. D. Hogan, and F. Merkt, Phys. Chem. Chem. Phys. 13, 19000 (2011).

[26] E. Vliegen and F. Merkt, J. Phys. B 39, L241 (2006).

[27] S. A. Meek, H. Conrad, and G. Meijer, Science 324, 1699 (2009).

[28] S. D. Hogan, J. A. Agner, F. Merkt, T. Thiele, S. Filipp, and A. Wallraff [Phys. Rev. Lett. (to be published)]. 